\title{
Optimal life-insurance selection and purchase within a market of several life-insurance providers
}

\author{
A.S. Mousa ${ }^{a, *}$, D. Pinheiro $^{b}$, A.A. Pinto ${ }^{c}$

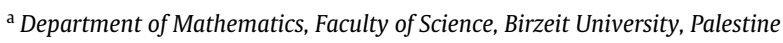 \\ ${ }^{\mathrm{b}}$ Department of Mathematics, Brooklyn College of the City University of New York, NY, USA \\ ${ }^{\mathrm{c}}$ LIAAD - INESC TEC and Department of Mathematics, Faculty of Science, University of Porto, Portugal
}

\section{A R T I C L E I N F O}

\section{Article history:}

Received June 2015

Received in revised form

October 2015

Accepted 13 January 2016

Available online 29 January 2016

\section{JEL classification:}

G22

G11

C61

Keywords:

Uncertain lifetime

Optimal consumption-investment

Life-insurance purchase and selection

Stochastic optimal control

Dynamic programming

\begin{abstract}
A B S T R A C T
We consider the problem faced by a wage-earner with an uncertain lifetime having to reach decisions concerning consumption and life-insurance purchase, while investing his savings in a financial market comprised of one risk-free security and an arbitrary number of risky securities whose prices are determined by diffusive linear stochastic differential equations. We assume that life-insurance is continuously available for the wage-earner to buy from a market composed of a fixed number of lifeinsurance companies offering pairwise distinct life-insurance contracts. We characterize the optimal consumption, investment and life-insurance selection and purchase strategies for the wage-earner with an uncertain lifetime and whose goal is to maximize the expected utility obtained from his family consumption, from the size of the estate in the event of premature death, and from the size of the estate at the time of retirement. We use dynamic programming techniques to obtain an explicit solution in the case of discounted constant relative risk aversion (CRRA) utility functions.
\end{abstract}

(c) 2016 Elsevier B.V. All rights reserved.

\section{Introduction}

We consider the case of a wage-earner whose lifetime is uncertain and faced with the problem of optimizing his decisions regarding consumption, investment and life-insurance selection and purchase during a random interval of time of the form $[0, \min \{\tau, T\}]$, where $T$ is a fixed instant of time in the future that can be seen as the retirement time of the wage-earner - and $\tau$ is a continuous and non-negative random variable representing the wage-earner's eventual time of death. We assume that life-insurance is available for the wage-earner to buy from a market composed of $K$ life-insurance companies and that the wage-earner's aim is to buy life-insurance in order to protect his family against the eventuality of his premature death. Each insurance company continuously offers life-insurance contracts, and the wage-earner buys life-insurance from insurance company $k$ by paying a premium insurance rate $p_{k}(t), k \in\{1,2, \ldots, K\}$. The insurance contracts to be considered herein are like term

\footnotetext{
* Corresponding author.

E-mail addresses: asaid@birzeit.edu (A.S. Mousa),

dpinheiro@brooklyn.cuny.edu (D. Pinheiro), aapinto@fc.up.pt (A.A. Pinto).
}

insurance, with an infinitesimally small term. If the wage-earner dies at time $\tau \leq T$ while buying insurance at the rate $p_{k}(t)$ from the $k$ th insurance company, then that insurance company pays, at the random time of death $\tau$, an amount

$Z_{k}(\tau)=\frac{p_{k}(\tau)}{\eta_{k}(\tau)}$

to his estate, where $\eta_{k}:[0, T] \rightarrow \mathbb{R}^{+}$is assumed to be a continuous and deterministic positive function, which we call the $k$ th insurance company premium-payout ratio. Such ratio specifies the life-insurance contract payout in the event of premature death and is regarded as fixed by the insurance company. Throughout this paper, we will assume that the insurance contracts offered by the $K$ insurance companies operating in the market are pairwise distinct in the sense that the corresponding premium-payout ratios are pairwise distinct for Lebesgue a.e. $t \in[0, T]$. Additionally, we will assume that every contract ends when the wage-earner dies or achieves retirement age, whichever happens first. Finally, we assume that the wage-earner invests the full amount of his savings in a financial market comprised of one risk-free security and a fixed number $N \geq 1$ of risky securities driven by a multi-dimensional Brownian motion.

Under the setup described above, the wage-earner is then faced with the problem of finding strategies that maximize the joint 
expected utility obtained from: his family consumption for all $t \leq$ $\min \{\tau, T\}$; his wealth at retirement date $T$ if he lives that long; and the value of his estate in the event of premature death. We prove that the optimal life-insurance selection and purchase strategy is to either buy no insurance from any company or else to buy an optimal (positive) amount of life-insurance from a single company: the one offering the smallest premium-payout ratio at each instant of time. The case where the optimal strategy is to buy no lifeinsurance at all is associated with wage-earners who hold large enough levels of wealth and close enough to retirement age, in such a way that paying for life-insurance contributes little towards an improvement of the utility gained from increasing the size of the estate in the event of premature death, while decreasing the overall joint expected utility of the wage-earner.

To address the problem described above, we will resort to dynamic programming techniques. Indeed, we should remark that the dynamic programming principle became one of the main techniques used to solve optimal control problems, after its initial development by Bellman $(1952,1953,1954)$ in the 1950s. Over the years, it has been extended to address stochastic optimal control problems (Bellman, 1958; Florentin, 1961, 1962; Kushner, 1962) with an increasing level of sophistication. The outcome of this technique is a backwards recursive relation for the value function associated with such problems and, under additional conditions, a nonlinear partial differential equation known as the Hamilton-Jacobi-Bellman (HJB) equation. For further details on this subject, we refer the reader to the textbooks by Fleming and Rishel (1975), Fleming and Soner (2006) and Yong and Zhou (1999).

A distinguishing feature of the problem under consideration here is that it presents a random time horizon. Indeed, there are currently several papers in topics related with Finance and Actuarial Science that can be formulated as stochastic optimal control problems with a random time horizon. One such example is the paper by Duarte et al. (2012), where a wage-earner with a random lifetime needs to decide about the optimal amount of life-insurance to buy from a single insurance company while investing his savings in a financial market. Shenab and Weib (2014) considered an optimal investment, consumption and lifeinsurance purchase problem for a wage-earner in a complete market with Brownian information and random parameters that are allowed to be unbounded. Previous works in this context were produced by Blanchet-Scalliet et al. (2008) and Pliska and Ye (2007), Ye (2006). The present study is also related to the models proposed by Yaari (1965), who introduced an optimal consumption problem for an individual with uncertain time of death within the setup of a pure deterministic investment environment, Hakansson (1969, 1971), who extended Yaari's model to a discretetime case with uncertainty including risky-assets, Merton (1969, 1971), who studied a continuous-time optimal consumption and investment problem (with no life-insurance purchase component), and Richard (1975), who combined the earlier approaches to obtain a continuous-time model for optimal consumption, investment and life-insurance purchase. However, we should reinforce that the problem considered herein looks into how a wage-earner should manage his portfolio of life-insurance policies and investment portfolio, a point of view which is somewhat complementary to the classical development of actuarial science, which has a heavier focus on ruin theory problems. This latter point of view has its roots on the seminal works leading to Cramér-Lundberg model introduced in the early 20th century (Lundberg, 1903, 1932; Cramér, 1969). The earlier results on ruin probability include the Lundberg inequality and Cramér-Lundberg approximation. However, a closed form solution for the ruin probability is known to be notoriously difficult to obtain, except for some very special cases. Moreover, since the assumption used in the Cramér-Lundberg model is too restrictive to be applicable in most cases, there has been intense work towards the development of more general and realistic models. Examples include the Sparre Andersen Model (Andersen, 1957), the Markov modulated risk model (Asmussen, 1989, 2000) and the diffusion-perturbed risk model (Gerber, 1970). Other approaches to extend the theory include studying the behavior of the insurer's surplus through an expected discounted penalty function, known as the Gerber-Shiu function (Powers, 1995; Gerber and Shiu, 1998), and the analysis of other relevant quantities such as the time of recovery from ruin as in Gerber (1990) and Egídio dos Reis (1993).

The practical relevance of the theoretical study presented herein lies on the attempt to provide a realistic model for the interaction between a wage-earner (who is simultaneously a saver and investor) and an insurance market composed of multiple (competing) insurance companies. For the purpose of our analysis, the wage-earner is assumed to be a completely rational agent whose ultimate aim is to maximize a given expected utility which encodes his preferences regarding consumption and wealth. As mentioned above, this leads us to optimal solutions whereby a typical wage-earner would, at any instant of time, buy lifeinsurance from the company offering the cheaper premium for the same level of protection, switching immediately to any competitor able to produce a better offer at a later instant in time. We believe that it may be interesting to extend this analysis by combining it with empirical data with the goal of assessing to what extent do real-life wage-earners (who are not fully rational in the most commonly used sense in economic theory) follow a reasonably similar pattern (allowing, eventually, for some potential delay in the contract switches due to real-life economic agents "bounded" rationality). Another possible extension for the results found herein is to consider more general financial markets, in order to study the influence that jumps, nonlinearities and regimeswitches may have on the optimal strategies regarding lifeinsurance selection and purchase. Such studies require a more general version of dynamic programming techniques as discussed in Azevedo et al. (2014).

This article is organized as follows. In Section 2 we describe the problem we propose to address. Namely, we introduce the underlying financial and insurance markets, the corresponding wealth process, and formulate the problem we wish to address in the framework of a stochastic optimal control with a random horizon. We then proceed to restate such problem as one with a fixed planning horizon, providing the corresponding dynamic programming principles and Hamilton-Jacobi-Bellman equation. We devote Section 3 to characterize the optimal strategies for the wage-earner under consideration here. An explicit solution for the case of discounted CRRA utility functions in obtained in Section 4. We conclude in Section 5.

\section{Problem formulation}

Throughout this section, we will introduce the underlying financial market available to the wage-earner, as well as the setup describing the insurance contracts under consideration herein. By the end of this section we will provide the definition of the wealth process for the wage-earner faced with the problem of optimizing his decisions regarding consumption, investment and life-insurance purchase during a given interval of time $[0, \min \{\tau, T\}]$.

\subsection{The financial market model}

Let $(\Omega, \mathcal{F}, P)$ be a complete probability space equipped with a filtration $\mathbb{F}=\left(\mathcal{F}_{t}\right)_{t \in[0, T]}$ given by the $P$-augmentation of the filtration generated by a standard $M$-dimensional Brownian motion $W(\cdot), \sigma(W(s): s \leq t)$ for $t \geq 0$. 
We consider a financial market consisting of one risk-free asset and finite number of risky-assets. Their respective prices $\left(S_{0}(t)\right)_{0 \leq t \leq T}$ and $\left(S_{n}(t)\right)_{0 \leq t \leq T}$, for $n=1, \ldots, N$, evolve according to the following stochastic differential equations:

$$
\begin{aligned}
\mathrm{d} S_{0}(t) & =r(t) S_{0}(t) \mathrm{d} t, \quad S_{0}(0)=s_{0}, \\
\mathrm{~d} S_{n}(t) & =\mu_{n}(t) S_{n}(t) \mathrm{d} t+S_{n}(t) \sum_{m=1}^{M} \sigma_{n m}(t) \mathrm{d} W_{m}(t), \\
S_{n}(0) & =s_{n}>0,
\end{aligned}
$$

where $W(t)=\left(W_{1}(t), \ldots, W_{M}(t)\right)^{T} \in \mathbb{R}^{M}, r(t)$ is the riskless interest rate, $\mu(t)=\left(\mu_{1}(t), \ldots, \mu_{N}(t)\right)^{T} \in \mathbb{R}^{N}$ is the vector of risky-assets appreciation rates, and $\sigma(t)=\left(\sigma_{n m}(t)\right)_{1 \leq n \leq N, 1 \leq m \leq M}$ is the $N \times M$ matrix of risky-assets volatilities. Each sub- $\sigma$ algebra $\mathcal{F}_{t}$ represents the information available to any given agent observing the financial market during the time interval $[0, t]$.

Assumption 2.1. The coefficients $r(t), \mu(t)$ and $\sigma(t)$ are deterministic continuous functions on the interval $[0, T]$. Additionally, the following conditions hold:

(i) the interest rate $r(t)$ is positive for all $t \in[0, T]$;

(ii) the matrix $\sigma(t)$ is such that $\sigma(t)(\sigma(t))^{T}$ is non-singular for Lebesgue almost all $t \in[0, T]$ and satisfies the following integrability condition

$$
\sum_{n=1}^{N} \sum_{m=1}^{M} \int_{0}^{T} \sigma_{n m}^{2}(t) \mathrm{d} t<\infty
$$

(iii) there exists an $\left(\mathcal{F}_{t}\right)_{0 \leq t \leq T}$-progressively measurable process $\pi(t) \in \mathbb{R}^{M}$, called the market price of risk, such that for Lebesgue-almost-every $t \in[0, T]$, the risk premium

$\alpha(t)=\left(\mu_{1}(t)-r(t), \ldots, \mu_{N}(t)-r(t)\right)^{T} \in \mathbb{R}^{N}$

is related to $\pi(t)$ by the equation

$\alpha(t)=\sigma(t) \pi(t)$ a.s.

and the following two conditions hold

$$
\begin{aligned}
& \int_{0}^{T}\|\pi(t)\|^{2} \mathrm{~d} t<\infty \text { a.s. } \\
& E\left[\exp \left(-\int_{0}^{T} \pi(s) \mathrm{d} W(s)-\frac{1}{2} \int_{0}^{T}\|\pi(s)\|^{2} \mathrm{~d} s\right)\right]=1 .
\end{aligned}
$$

The existence of the market price of risk $\pi(t)$ ensures the absence of arbitrage opportunities in the financial market defined above. Note also that the conditions on the matrix $\sigma$ above do not imply market completeness. See Karatzas and Shreve (1998) for further details on market viability and completeness.

\subsection{The life-insurance market model}

The wage-earner is alive at time $t=0$ and his lifetime is a nonnegative continuous random variable $\tau$ defined on the probability space $(\Omega, \mathcal{F}, P)$.

Assumption 2.2. The random variable $\tau$ is independent of the filtration $\mathbb{F}$ and has a distribution function $G^{-}:[0, \infty) \rightarrow[0,1]$ with density $g:[0, \infty) \rightarrow \mathbb{R}^{+}$such that

$G^{-}(t)=P(\tau \leq t)=\int_{0}^{t} g(s) \mathrm{d} s$.

Recall that the survival function $G^{+}:[0, \infty) \rightarrow[0,1]$ is defined as the probability for the wage-earner to survive past time $t$, i.e.

$G^{+}(t)=P(\tau>t)=1-G^{-}(t)$.
We shall also make use of the hazard rate function, the conditional, instantaneous death rate for the wage-earner surviving past time $t$, that is

$\lambda(t)=\lim _{\delta t \rightarrow 0} \frac{P(t<\tau \leq t+\delta t \mid \tau>t)}{\delta t}=\frac{g(t)}{G^{+}(t)}$.

Throughout this paper, we will suppose that the hazard rate function $\lambda:[0, \infty) \rightarrow \mathbb{R}^{+}$is a continuous and deterministic function such that

$\int_{0}^{\infty} \lambda(t) \mathrm{d} t=\infty$

The concepts introduced above are standard in the context of Reliability Theory and Actuarial Science (see, e.g. Klugman et al., 2012, Bowers et al., 1997).

As mentioned in the introduction, the life-insurance market under consideration here is composed of $K$ insurance companies, with each insurance company continuously offering life-insurance contracts. The wage-earner buys life-insurance from insurance company $k$ by paying a premium insurance rate $p_{k}(t)$ for each $k=$ $1,2, \ldots, K$. The insurance contracts are like term insurance, with an infinitesimally small term. If the wage-earner dies at time $\tau \leq T$ while buying insurance at the rate $p_{k}(t)$ from the $k$ th insurance company, then that insurance company pays an amount

$Z_{k}(\tau)=\frac{p_{k}(\tau)}{\eta_{k}(\tau)}$

to his estate, where $\eta_{k}:[0, T] \rightarrow \mathbb{R}^{+}$is the $k$ th insurance company premium-payout ratio.

Assumption 2.3. For every $k \in\{1, \ldots, K\}$, the $k$ th insurance company premium-payout ratio $\eta_{k}(t)$ is a continuous and deterministic function. Additionally, we will assume that the $K$ insurance companies under consideration here offer pairwise distinct contracts in the sense that $\eta_{k_{1}}(t) \neq \eta_{k_{2}}(t)$ for every $k_{1} \neq$ $k_{2}$ and Lebesgue-almost-every $t \in[0, T]$.

As a consequence of Assumption 2.3, we have that the $K \times K$ symmetric matrix $\eta(t)^{T} \eta(t)$, where $\eta(t)=\left(\eta_{1}(t), \eta_{2}(t), \ldots\right.$, $\left.\eta_{K}(t)\right)^{T} \in\left(\mathbb{R}^{+}\right)^{K}$, is non-singular for Lebesgue almost-every $t \in$ $[0, T]$.

The contract ends when the wage-earner dies or achieves retirement age, whichever happens first. Therefore, the wageearner's total legacy to his estate in the event of a premature death at time $\tau \leq T$ is given by

$Z(\tau)=X(\tau)+\sum_{k=1}^{K} \frac{p_{k}(\tau)}{\eta_{k}(\tau)}$

where $X(t)$ denotes the wage-earner's wealth at time $t \in[0, T]$.

\subsection{The wealth process}

The wage-earner receives income $i(t)$ at a continuous rate during the period $[0, \min \{\tau, T\}]$, i.e. the income will be terminated either by his death or his retirement, whichever happens first.

Assumption 2.4. The income function $i:[0, T] \rightarrow \mathbb{R}_{0}^{+}$is a deterministic Borel-measurable function satisfying the integrability condition:

$\int_{0}^{T} i(t) \mathrm{d} t<\infty$.

The consumption process $(c(t))_{0 \leq t \leq T}$ is a $\left(\mathcal{F}_{t}\right)_{0 \leq t \leq T}$-progressively measurable non-negative process satisfying the following 
integrability condition for the investment horizon $T>0$ :

$\int_{0}^{T} c(t) \mathrm{d} t<\infty$ a.s.

We assume also that for all $k=1,2, \ldots, K$, the $k$ th company premium insurance rate $\left(p_{k}(t)\right)_{0 \leq t \leq T}$ is non-negative a $\left(\mathcal{F}_{t}\right)_{0 \leq t \leq T^{-}}$ predictable process, i.e. $p_{k}(t)$ is measurable with respect to the smallest $\sigma$-algebra on $\mathbb{R}_{0}^{+} \times \Omega$ such that all left-continuous and adapted processes are measurable.

For each $n=0,1, \ldots, N$ and $t \in[0, T]$, let $\theta_{n}(t)$ denote the fraction of the wage-earner's wealth allocated to the asset $S_{n}$ at time $t$. The wage-earner portfolio process is then given by $\Theta(t)=$ $\left(\theta_{0}(t), \theta_{1}(t), \ldots, \theta_{N}(t)\right)^{T} \in \mathbb{R}^{N+1}$, where

$\sum_{n=0}^{N} \theta_{n}(t)=1, \quad 0 \leq t \leq T$.

We assume that the portfolio process is $\left(\mathcal{F}_{t}\right)_{0 \leq t \leq T}$-progressively measurable and that for the fixed investment horizon $T>0$ we have that

$\int_{0}^{T}\|\Theta(t)\|^{2} \mathrm{~d} t<\infty$ a.s.

where $\|\cdot\|$ denotes the Euclidean norm in $\mathbb{R}^{N+1}$.

The wealth process $X(t), t \in[0, \min \{\tau, T\}]$, is then defined by

$$
\begin{aligned}
X(t)= & x+\int_{0}^{t}\left(i(s)-c(s)-\sum_{k=1}^{K} p_{k}(s)\right) \mathrm{d} s \\
& +\sum_{n=0}^{N} \int_{0}^{t} \frac{\theta_{n}(s) X(s)}{S_{n}(s)} \mathrm{d} S_{n}(s),
\end{aligned}
$$

where $x$ is the wage-earner's initial wealth. This last equation can be rewritten in differential form as

$$
\begin{aligned}
\mathrm{d} X(t)= & \left(i(t)-c(t)-\sum_{k=1}^{K} p_{k}(t)+\left(\theta_{0}(t) r(t)\right.\right. \\
& \left.\left.+\sum_{n=1}^{N} \theta_{n}(t) \mu_{n}(t)\right) X(t)\right) \mathrm{d} t \\
& +\sum_{n=1}^{N} \theta_{n}(t) X(t) \sum_{m=1}^{M} \sigma_{n m}(t) \mathrm{d} W_{m}(t),
\end{aligned}
$$

where $0 \leq t \leq \min \{\tau, T\}$.

Using relation (3), we can always write $\theta_{0}(t)$ in terms of $\theta_{1}(t), \ldots, \theta_{N}(t)$. From now on, we will define the portfolio process in terms of the wage-earner reduced portfolio process $\theta(t) \in \mathbb{R}^{N}$, given by

$\theta(t)=\left(\theta_{1}(t), \theta_{2}(t), \ldots, \theta_{N}(t)\right)^{T} \in \mathbb{R}^{N}$.

We shall represent the wage-earner life-insurance purchase rate as a vector

$p(t)=\left(p_{1}(t), p_{2}(t), \ldots, p_{K}(t)\right)^{T} \in\left(\mathbb{R}_{0}^{+}\right)^{K}$,

where for each $k \in\{1,2, \ldots, K\}$, the quantity $p_{k}(t)$ denotes the life-insurance purchase rate from the $k$ th insurance company at time $t \in[0, \min \{\tau, T\}]$. Note that a zero component in $p(t)$ represents the absence of any life-insurance contract between the wage-earner and a certain insurance company.

\subsection{The optimal control problem}

The wage-earner is faced with the problem of finding strategies that maximize the expected utility obtained from: his family consumption for all $t \leq \min \{\tau, T\}$; his wealth at retirement date $T$ if he lives that long; and the value of his estate in the event of premature death.

This problem can be formulated by means of optimal control theory. The wage-earner goal is to maximize some cost functional subject to the (stochastic) dynamics of the state variable, i.e. the dynamics of the wealth process $X(t)$ given by (4); constraints on the control variables, i.e. the consumption process $c(t)$, the premium insurance rates $p(t)$ and the portfolio process $\theta(t)$; and boundary conditions on the state variables.

Let us denote by $\mathcal{A}(0, x)$ the set of all admissible decision strategies, i.e. all admissible choices for the control variables $v=(c(\cdot), \theta(\cdot), p(\cdot))$. The dependence of $\mathcal{A}(0, x)$ on $x$ denotes the restriction imposed on the wealth process by the boundary condition $X(0)=x$.

The wage-earner's problem can then be restated as follows: find a strategy $v=(c(\cdot), \theta(\cdot), p(\cdot)) \in \mathcal{A}(0, x)$ which maximizes the expected utility

$$
\begin{aligned}
& E_{0, x}\left[\int_{0}^{\tau \wedge T} U(s, c(s)) \mathrm{d} s+B(\tau, Z(\tau)) I_{[0, T]}(\tau)\right. \\
& \left.\quad+W(X(T)) I_{(T,+\infty)}(\tau)\right],
\end{aligned}
$$

where $\tau \wedge T=\min \{\tau, T\}, I_{A}$ denotes the indicator function of the set $A, U(t, \cdot)$ is the utility function describing the wage-earner's family preferences regarding consumption at some instant of time $t \in[0, T], W(\cdot)$ is the utility function for the terminal wealth at retirement time $T$, and $B(t, \cdot)$ is the utility function for the size of the wage-earners's legacy at some time $t \in[0, T]$ as given in (2).

Assumption 2.5. The utility functions $U:[0, T] \times \mathbb{R}_{0}^{+} \rightarrow \mathbb{R}_{0}^{+}$ and $B:[0, T] \times \mathbb{R}_{0}^{+} \rightarrow \mathbb{R}_{0}^{+}$are twice differentiable, strictly increasing and strictly concave functions on their second variable, and $W: \mathbb{R}_{0}^{+} \rightarrow \mathbb{R}_{0}^{+}$is a twice differentiable, strictly increasing and strictly concave function.

In Section 4 we will specialize our analysis to the case where the wage-earner's preferences are described by discounted CRRA utility functions.

\subsection{The stochastic optimal control problem}

We use the techniques introduced in Pliska and Ye (2007), Ye (2006) to restate the stochastic optimal control problem formulated above as one with a fixed planning horizon. We then state a dynamic programming principle and the corresponding HJB equation.

Denote by $\mathcal{A}(t, x)$ the set of admissible decision strategies $v=(c(\cdot), \theta(\cdot), p(\cdot))$ for the dynamics of the wealth process with boundary condition $X(t)=x$. For any $v \in \mathcal{A}(t, x)$ we define

$$
\begin{aligned}
J(t, x ; v)= & E_{t, x}\left[\int_{t}^{\tau \wedge T} U(s, c(s)) \mathrm{d} s+B(\tau, Z(\tau)) I_{[0, T]}(\tau)\right. \\
& \left.+W\left(X_{t, x}^{v}(T)\right) I_{(T,+\infty)}(\tau) \mid \tau>t, \mathcal{F}_{t}\right]
\end{aligned}
$$

where $X_{t, x}^{v}(s) \geq 0$ denotes the wealth process starting from $x$ at time $t \leq s$ under the choice of the control $v \in \mathcal{A}(t, x)$. That is, $X_{t, x}^{v}(s)$ is the solution of the stochastic differential equation (5) with initial condition $X(t)=x$. 
For every $0 \leq t \leq s$, let $G^{+}(s, t)$ denote the conditional probability for the wage-earner to be alive at time $s$ conditional upon being alive at time $t \leq s$, given by

$G^{+}(s, t)=P(\{\tau>s\} \mid\{\tau>t\})$

and $G^{-}(s, t)$ denote the conditional probability for the wageearner time of death to occur at time $s$ conditional upon being alive at time $t \leq s$, given by

$G^{-}(s, t)=P(\{\tau \leq s\} \mid\{\tau>t\})$.

Let $g^{-}(s, t)$ denote the density function associated with the conditional distribution function $G^{-}(s, t)$, given by

$g^{-}(s, t)=\frac{\mathrm{d}}{\mathrm{d} t} G^{-}(s, t)$.

The following lemma enables the transformation of the optimal control problem above to an equivalent one with a fixed planning horizon. See Ye (2006) for its proof.

Lemma 2.6. Suppose that Assumptions 2.1-2.5 hold. If the random variable $\tau$ is independent of the filtration $\mathbb{F}$, then

$$
\begin{aligned}
J(t, x ; v)= & E_{t, x}\left[\int _ { t } ^ { T } \left(G^{+}(s, t) U(s, c(s))\right.\right. \\
& \left.+g(s, t) B(s, Z(s))) \mathrm{d} s+G^{+}(T, t) W(X(T)) \mid \mathcal{F}_{t}\right],
\end{aligned}
$$

where the conditional probabilities $G^{+}(s, t)$ and $g^{-}(s, t)$ are as given in (7) and (8), respectively.

The transformation to a fixed planning horizon provided above can be given the following interpretation: a wage-earner facing unpredictable death acts as if he will live until time $T$, but with a subjective rate of time preferences equal to his "force of mortality" for the consumption of his family and his terminal wealth.

Note that the optimal control problem (6) can now be restated in dynamic programming form as

$V(t, x)=\sup _{v \in \mathcal{A}(t, x)} J(t, x ; v)$.

Using the previous lemma, one can state a dynamic programming principle, obtaining a recursive relationship for the value function $V(t, x)$. See Ye (2006) for the proof.

Lemma 2.7 (Dynamic Programming Principle). Suppose that Assumptions 2.1-2.5 hold. For $0 \leq t<s<T$, the maximum expected utility $V(t, x)$ satisfies the recursive relation

$$
\begin{aligned}
V(t, x)= & \sup _{v \in \mathcal{A}(t, x)} E\left[\exp \left(-\int_{t}^{s} \lambda(u) \mathrm{d} u\right) V\left(s, X_{t, x}^{v}(s)\right)\right. \\
& +\int_{t}^{s}\left(G^{+}(u, t) U(u, c(u))\right. \\
& \left.\left.+g(u, t) B\left(u, Z_{t, x}^{v}(u)\right)\right) \mathrm{d} u \mid \mathcal{F}_{t}\right] .
\end{aligned}
$$

The dynamic programming principle enables us to state a HJB equation, a second-order partial differential equation whose solution, whenever it exists, is the value function of the optimal control problem under consideration here. The techniques used in the derivation of the HJB equation and the proof of the next theorem follow closely those in Fleming and Soner (2006), Ye (2006) and Yong and Zhou (1999).
Theorem 2.8 (Hamilton-Jacobi-Bellman Equation). Suppose that Assumptions 2.1-2.5 hold and that the value function $V$ is of class $C^{1,2}([0, T] \times \mathbb{R}, \mathbb{R})$. Then $V$ satisfies the Hamilton-Jacobi-Bellman equation

$$
\left\{\begin{array}{l}
V_{t}(t, x)-\lambda(t) V(t, x)+\sup _{(c, \theta, p) \in \mathbb{R}^{N+1} \times\left(\mathbb{R}_{0}^{+}\right)^{K}} \mathcal{H}(t, x ; c, \theta, p)=0 \\
V(T, x)=W(x),
\end{array}\right.
$$

where the Hamiltonian function $\mathscr{H}$ is given by

$$
\begin{aligned}
\mathcal{H}(t, x ; v)= & \left(i(t)-c(t)-\sum_{k=1}^{K} p_{k}\right. \\
& \left.+\left(r(t)+\sum_{n=1}^{N} \theta_{n}\left(\mu_{n}(t)-r(t)\right)\right) x\right) V_{x}(t, x) \\
& +\frac{x^{2}}{2} \sum_{m=1}^{M}\left(\sum_{n=1}^{N} \theta_{n} \sigma_{n m}(t)\right)^{2} V_{x x}(t, x) \\
& +U(t, c)+\lambda(t) B\left(t, x+\sum_{k=1}^{K} \frac{p_{k}}{\eta_{k}(t)}\right) .
\end{aligned}
$$

Moreover, an admissible strategy $v^{*}=\left(c^{*}(\cdot), \theta^{*}(\cdot), p^{*}(\cdot)\right) \in$ $\mathcal{A}(t, x)$ whose corresponding wealth is $X^{*}$ is optimal if and only if for a.e. $s \in[t, T]$ and $P$-a.s. we have

$V_{t}\left(s, X^{*}(s)\right)-\lambda(s) V\left(s, X^{*}(s)\right)+\mathscr{H}\left(s, X^{*}(s) ; v^{*}\right)=0$.

\section{The optimal strategies}

The second part of Theorem 2.8 provides a strategy for the computation of the optimal insurance selection and purchase, portfolio and consumption strategies for the wage-earner with uncertain lifetime under consideration herein.

For each $t \in[0, T]$, let $U_{x}(t, \cdot)$ and $B_{x}(t, \cdot)$ denote, respectively, the derivatives of the utility functions $U(t, \cdot)$ and $B(t, \cdot)$ with respect to their second arguments. Since both $U(t, \cdot)$ and $B(t, \cdot)$ are strictly concave with respect to their second arguments, the corresponding derivatives are invertible. Hence, we define $I_{1}$ : $[0, T] \times \mathbb{R}_{0}^{+} \rightarrow \mathbb{R}_{0}^{+}$and $I_{2}:[0, T] \times \mathbb{R}_{0}^{+} \rightarrow \mathbb{R}_{0}^{+}$to be the (unique) functions such that

$I_{1}\left(t, U_{x}(t, x)\right)=x \quad$ and $\quad U_{x}\left(t, I_{1}(t, x)\right)=x$

and

$I_{2}\left(t, B_{x}(t, x)\right)=x \quad$ and $\quad B_{x}\left(t, I_{2}(t, x)\right)=x$

for every $t \in[0, T]$ and $x \in \mathbb{R}_{0}^{+}$.

The next result provides a characterization for the optimal strategies in terms of the value function and its derivatives.

Theorem 3.1. Suppose that Assumptions 2.1-2.5 hold and that the value function $V$ is of class $C^{1,2}([0, T] \times \mathbb{R}, \mathbb{R})$. Then the Hamiltonian function $\mathscr{H}$ given in the statement of Theorem 2.8 has a unique maximum $v^{*}=\left(c^{*}(\cdot), \theta^{*}(\cdot), p^{*}(\cdot)\right) \in \mathcal{A}(t, x)$. Moreover, the optimal strategies are given by

$c^{*}(t, x)=I_{1}\left(t, V_{x}(t, x)\right)$

$\theta^{*}(t, x)=-\frac{V_{x}(t, x)}{x V_{x x}(t, x)} \xi \alpha(t)$

and, for each $k \in\{1,2, \ldots, K\}$, we have that

$p_{k}^{*}(t, x)=\left\{\begin{array}{l}\max \left\{0,\left[I_{2}\left(t, \eta_{k}(t)(\lambda(t))^{-1} V_{x}(t, x)\right)-x\right] \eta_{k}(t)\right\}, \\ \quad \text { if } k=k^{*}(t) \\ 0, \quad \text { otherwise, }\end{array}\right.$ 
where

$k^{*}(t)=\underset{k \in\{1,2, \ldots, K\}}{\arg \min }\left\{\eta_{k}(t)\right\}$

and $\xi$ denotes the non-singular square matrix given by $\left(\sigma \sigma^{T}\right)^{-1}$ and $\alpha(t)$ is as given in (1).

Proof. Using the second part of Theorem 2.8, an optimal admissible strategy $v^{*}=\left(c^{*}, \theta^{*}, p^{*}\right) \in \mathcal{A}(t, x)$ with wealth process $X^{*}$ must satisfy (10). Therefore, $v^{*}$ must be such that $\mathscr{H}$ attains its maximum value. We start by remarking that the condition determining the maximum for $\mathscr{H}$ decouples into three independent conditions:

$$
\begin{aligned}
& \sup _{(c, \theta, p) \in \mathbb{R}^{N+1} \times\left(\mathbb{R}_{0}^{+}\right)^{K}} \mathcal{H}(t, x ; v) \\
& =\sup _{c \in \mathbb{R}}\left\{U(t, c)-c V_{x}(t, x)\right\}+r(t) x V_{x}(t, x) \\
& +\sup _{p \in\left(\mathbb{R}_{0}^{+}\right)^{K}}\left\{\lambda(t) B\left(t, x+\sum_{k=1}^{K} \frac{p_{k}}{\eta_{k}(t)}\right)-V_{x}(t, x) \sum_{k=1}^{K} p_{k}\right\} \\
& +i(t) V_{x}(t, x)+\sup _{\theta \in \mathbb{R}^{N}}\left\{\frac{x^{2}}{2} \sum_{m=1}^{M}\left(\sum_{n=1}^{N} \theta_{n} \sigma_{n m}(t)\right)^{2}\right. \\
& \left.\times V_{x x}(t, x)+\sum_{n=1}^{N} \theta_{n}\left(\mu_{n}(t)-r(t)\right) x V_{x}(t, x)\right\} .
\end{aligned}
$$

Therefore, it is enough to study the variation of $\mathscr{H}$ with respect to each one of the variables $c, \theta$ and $p$ independently. We deal with the unconstrained optimization problems associated with $c$ and $\theta$ first. Computing the first-order conditions with respect to $c$ and $\theta$ we obtain, respectively, the following $N+1$ conditions:

$$
\begin{aligned}
-V_{x}(t, x)+U_{x}\left(t, c^{*}\right) & =0 \\
x V_{x}(t, x) \alpha+x^{2} V_{x x}(t, x) \sigma \sigma^{T} \theta^{*} & =0_{\mathbb{R}^{N}},
\end{aligned}
$$

where $\alpha$ denotes the risk premium given in (1) and $0_{\mathbb{R}^{N}}$ denotes the origin of $\mathbb{R}^{N}$. Resorting to the inverse functions introduced before the statement of Theorem 3.1, we can solve Eq. (12) for the control variables and get

$$
\begin{aligned}
& c^{*}(t, x)=I_{1}\left(t, V_{x}(t, x)\right) \\
& \theta^{*}(t, x)=-\frac{V_{x}(t, x)}{x V_{x x}(t, x)} \xi \alpha(t) .
\end{aligned}
$$

To solve the constrained optimization problem associated with the variable $p \in\left(\mathbb{R}_{0}^{+}\right)^{K}$, we resort to the Kuhn-Tucker conditions. Namely, we look for a solution $\left(p_{1}(t, x), \ldots, p_{K}(t, x)\right.$, $\left.\mu_{1}(t, x), \ldots, \mu_{K}(t, x)\right)$ to the following set of equalities and inequalities:

$$
\begin{aligned}
\frac{\lambda(t)}{\eta_{k}(t)} B_{x}\left(t, x+\sum_{k=1}^{K} \frac{p_{k}}{\eta_{k}(t)}\right)-V_{x}(t, x) & =-\mu_{k}, \\
p_{k} & \geq 0, \\
\mu_{k} & \geq 0, \quad k=1,2, \ldots, K \\
p_{k} \mu_{k} & =0 .
\end{aligned}
$$

We start by noting that for $k_{1} \neq k_{2}$, if we have $\mu_{k_{1}}(t, x)=\mu_{k_{2}}(t, x)$ for some $(t, x) \in[0, T] \times \mathbb{R}$, one must have that $\eta_{k_{1}}(t)=\eta_{k_{2}}(t)$. Thus, relying on the assumption that all insurance companies offer pairwise distinct contracts, we obtain that for every $k_{1}, k_{2} \in$ $\{1,2, \ldots, K\}$ such that $k_{1} \neq k_{2}$ and every $x \in \mathbb{R}, \mu_{k_{1}}(t, x) \neq$ $\mu_{k_{2}}(t, x)$ for Lebesgue a.e. $t \in[0, T]$. In particular, we obtain that for every $x \in \mathbb{R}$ and Lebesgue a.e. $t \in[0, T]$, there is at most one $k \in\{1,2, \ldots, K\}$ such that $\mu_{k}(t, x)=0$. Therefore, for Lebesgue a.e. $t \in[0, T]$, there is at most one $k \in\{1,2, \ldots, K\}$ such that $p_{k}(t, x) \neq 0$.

Using once again the first identity in (13), we get that

$\eta_{k_{1}}(t)\left(V_{x}(t, x)-\mu_{k_{1}}\right)=\eta_{k_{2}}(t)\left(V_{x}(t, x)-\mu_{k_{2}}\right)$.

As a consequence of the identity above, we conclude that if $\mu_{k_{1}}(t, x)>\mu_{k_{2}}(t, x)$ for $(t, x) \in[0, T] \times \mathbb{R}$, then $\eta_{k_{1}}(t)>\eta_{k_{2}}(t)$. Furthermore, if for some $t \in[0, T]$ we have $\mu_{k_{1}}(t, x)=0$, then $\eta_{k_{1}}(t)<\eta_{k_{2}}(t)$ for every $k_{2} \in\{1,2, \ldots, K\}$ such that $k_{1} \neq k_{2}$.

From this point onwards, let $k^{*}(t)$ be as given in (11). Then, either $p_{k}(t, x)=0$ for every $k \in\{1,2, \ldots, K\}$ or else $p_{k^{*}(t)}(t, x)>0$ is a solution to

$\frac{\lambda(t)}{\eta_{k^{*}(t)}(t)} B_{x}\left(t, x+\frac{p_{k^{*}(t)}}{\eta_{k^{*}(t)}(t)}\right)=V_{x}(t, x)$,

yielding

$p_{k}^{*}(t, x)=\left\{\begin{array}{l}\max \left\{0,\left[I_{2}\left(t, \frac{\eta_{k}(t) V_{x}(t, x)}{\lambda(t)}\right)-x\right] \eta_{k}(t)\right\}, \\ \text { if } k=k^{*}(t) \\ 0 \quad \text { otherwise. }\end{array}\right.$

Computing the second derivative with respect to each variable (the Hessian matrix in the case of variable $\theta$ ), we obtain

$$
\begin{aligned}
\mathscr{H}_{c c}\left(t, x ; v^{*}\right) & =U_{c c}\left(t, c^{*}\right) \\
\mathscr{H}_{p_{k_{1}} p_{k_{2}}}\left(t, x ; v^{*}\right)= & \frac{\lambda(t)}{\eta_{k_{1}}(t) \eta_{k_{2}}(t)} B_{x x}\left(t, x+\frac{p_{k^{*}(t)}^{*}}{\eta_{k^{*}(t)}(t)}\right), \\
& k_{1}, k_{2}=1, \ldots, K \\
\mathcal{H}_{\theta \theta}\left(t, x ; v^{*}\right)= & x^{2} V_{x x}(t, x) \sigma \sigma^{T} .
\end{aligned}
$$

Optimality of $c^{*}$ and $p^{*}$ follows from strict concavity of the functions $U$ and $B$ with respect to their second variables, which makes the first-order conditions and the Kuhn-Tucker conditions not only necessary, but also sufficient. In what concerns the optimization with respect to $\theta$, we need to see that $\mathscr{H}_{\theta \theta}\left(t, x ; v^{*}\right)$ is negative definite. Recall that $\sigma \sigma^{T}$ is assumed to be non-singular and, thus, positive definite. Moreover, note that $V_{x x}(t, x)$ must be negative: if $V_{x x}(t, x)$ was positive, then $\mathscr{H}$ would not be bounded above, and as a consequence of the HJB equation, either $V_{t}(t, x)$ or $V(t, x)$ would have to be infinity, contradicting the smoothness assumption on $V$. Therefore, $\mathscr{H}_{\theta \theta}$ is negative definite and $\mathscr{H}$ has a unique regular interior maximum.

Remark 3.2. As time changes, the insurance company picked by the wage-earner may change, and this happens according to the relative values of the functions $\eta_{1}, \eta_{2}, \ldots, \eta_{K}$ : the client always picks the insurance company offering the lowest $\eta_{k}$, if any. However, for a set of times with zero Lebesgue measure, one may have equality of at least a pair of premium-payout ratios, leading to some indeterminacy on the choice of the index $k^{*}(t)$ in these instants of time. Nevertheless, such instants of time correspond to switching times between two alternative insurance companies, and such indeterminacy has no impact on the behavior of the optimal strategies of Theorem 3.1.

Remark 3.3. A final comment is in order regarding the cases where the optimal strategy is to avoid buying any life-insurance. We prove in Theorem 3.1 that the optimal strategy is to buy insurance from at most one insurance company at every instant of time. In Duarte et al. (2012), where the case of a single insurance company was considered, it was observed that for wage-earners with sufficiently large amount of wealth and age sufficiently close to retirement, the optimal strategy would be, if possible from a practical point of view, to take a short position on its own life-insurance contract. Since we work here under the more 
realistic assumption of non-negative life-insurance purchase, then such special cases correspond to optimal life-insurance purchase strategies under which the wage-earner buys no life-insurance at all.

\section{The family of discounted CRRA utilities}

In this section we describe the special case where the wageearner has the same discounted CRRA utility functions for the consumption of his family, the size of his estate in the event of premature death and his terminal wealth. Henceforth, we assume that the utility functions are given by

$U(t, c)=\mathrm{e}^{-\rho t} \frac{c^{\gamma}}{\gamma}, \quad W(X)=\mathrm{e}^{-\rho T} \frac{X^{\gamma}}{\gamma}$ and

$B(t, Z)=\mathrm{e}^{-\rho t} \frac{Z^{\gamma}}{\gamma}$,

where the risk aversion parameter $\gamma$ is such that $\gamma<1, \gamma \neq 0$ and the discount rate $\rho$ is positive.

\subsection{The optimal strategies}

Using the optimality criteria provided in Theorem 3.1, we obtain the following optimal strategies for discounted CRRA utility functions.

Proposition 4.1. Suppose that Assumptions 2.1-2.4 hold. Let $\xi$ denote the non-singular square matrix given by $\left(\sigma \sigma^{T}\right)^{-1}$. The optimal strategies in the case of discounted CRRA utility functions are given by

$c^{*}(t, x)=\frac{1}{e(t)}(x+b(t))$

$\theta^{*}(t, x)=\frac{1}{1-\gamma} \frac{x+b(t)}{x} \xi \alpha(t)$,

$p_{k}^{*}(t, x)=\left\{\begin{array}{l}\max \left\{0, \eta_{k}(t)((D(t)-1) x+D(t) b(t))\right\}, \\ \text { if } k=k^{*}(t) \\ 0 \text { otherwise, }\end{array}\right.$

where

$$
\begin{aligned}
b(t)= & \int_{t}^{T} i(s) \exp \left(-\int_{t}^{s}\left(r(v)+\eta_{k^{*}(v)}(v)\right) \mathrm{d} v\right) \mathrm{d} s \\
D(t)= & \frac{1}{e(t)}\left(\frac{\lambda(t)}{\eta_{k^{*}(t)}}\right)^{1 /(1-\gamma)} \\
e(t)= & \exp \left(-\int_{t}^{T} H(v) \mathrm{d} v\right) \\
& +\int_{t}^{T} \exp \left(-\int_{t}^{s} H(v) \mathrm{d} v\right) L(s) \mathrm{d} s \\
H(t)= & \frac{\lambda(t)+\rho}{1-\gamma}-\frac{\gamma}{1-\gamma}\left(r(t)+\eta_{k^{*}(t)}(t)\right)-\frac{\gamma}{(1-\gamma)^{2}} \Sigma(t) \\
L(t)= & 1+\left(\frac{\lambda(t)}{\left(\eta_{k^{*}(t)}(t)\right)^{\gamma}}\right)^{1 /(1-\gamma)} \\
\Sigma(t)= & \alpha^{T}(t) \xi \alpha(t)-\frac{1}{2}\left\|\sigma^{T} \xi \alpha(t)\right\|^{2} .
\end{aligned}
$$

Proof. Assume that the utility functions $U, B$ and $W$ are as given in (15). Using the results of Theorem 3.1, we obtain that the optimal strategies depending on the value function $V$ are given by

$c^{*}(t, x)=\left(\mathrm{e}^{\rho t} V_{x}(t, x)\right)^{-1 /(1-\gamma)}$

$$
\theta^{*}(t)=-\frac{V_{x}(t, x)}{x V_{x x}(t, x)} \xi \alpha(t)
$$

$$
p_{k}^{*}(t, x)
$$

$$
=\left\{\begin{array}{l}
\max \left\{0,\left(\left(\frac{\eta_{k}(t) \mathrm{e}^{\rho t} V_{x}(t, x)}{\lambda(t)}\right)^{-1 /(1-\gamma)}-x\right) \eta_{k}(t)\right\}, \\
\quad \text { if } k=k^{*}(t) \\
0 \quad \text { otherwise. }
\end{array}\right.
$$

We are now going to find an explicit solution for the HJB equation (9). We substitute $c, \theta$ and $p_{k}$ in the HJB equation by the optimal strategies in (16) and combine similar terms to arrive at the following partial differential equation

$$
\begin{aligned}
& V_{t}(t, x)-\lambda(t) V(t, x)+\left(i(t)+\left(r(t)+\eta_{k^{*}(t)}(t)\right) x\right) V_{x}(t, x) \\
& -\Sigma(t) \frac{\left(V_{x}(t, x)\right)^{2}}{V_{x x}(t, x)}+\frac{1-\gamma}{\gamma} \mathrm{e}^{-\rho t /(1-\gamma)} L(t) \\
& \quad \times\left(V_{x}(t, x)\right)^{-\gamma /(1-\gamma)}=0,
\end{aligned}
$$

where $\Sigma(t)$ and $L(t)$ are as given in the statement of this proposition, and the terminal condition is given by

$V(T, x)=W(x)$.

We consider an ansatz of the form

$V(t, x)=\frac{a(t)}{\gamma}(x+b(t))^{\gamma}$,

and substitute it in (17) so that $a(t)$ and $b(t)$ are determined by the differential equation

$$
\begin{aligned}
& \frac{1}{\gamma} \frac{\mathrm{d} a(t)}{\mathrm{d} t}+\frac{a(t)}{x+b(t)} \frac{\mathrm{d} b(t)}{\mathrm{d} t}-\frac{\lambda(t)}{\gamma} a(t) \\
& +\frac{\left(i(t)+\left(r(t)+\eta_{k^{*}(t)}(t)\right) x\right)}{x+b(t)} a(t) \\
& \quad+\Sigma(t) \frac{a(t)}{1-\gamma}+\frac{1-\gamma}{\gamma} \mathrm{e}^{-\rho t /(1-\gamma)} L(t)(a(t))^{-\gamma /(1-\gamma)}=0 .
\end{aligned}
$$

Note now that the previous differential equation and the terminal condition (18) decouple into two independent boundary value problems for $a(t)$ and $b(t)$ which are given, respectively, by

$$
\begin{aligned}
& \frac{1}{\gamma} \frac{\mathrm{d} a(t)}{\mathrm{d} t}+\left(r(t)+\eta_{k^{*}(t)}(t)-\frac{\lambda(t)}{\gamma}+\frac{\Sigma(t)}{1-\gamma}\right) a(t) \\
& +\frac{1-\gamma}{\gamma} \mathrm{e}^{-\rho t /(1-\gamma)} L(t)(a(t))^{-\gamma /(1-\gamma)}=0 \\
& a(T)=\mathrm{e}^{-\rho T},
\end{aligned}
$$

and

$$
\begin{aligned}
& \frac{\mathrm{d} b(t)}{\mathrm{d} t}-\left(r(t)+\eta_{k^{*}(t)}(t)\right) b(t)+i(t)=0 \\
& b(T)=0 .
\end{aligned}
$$

To find a solution to the boundary value problem (20), we write $a(t)$ in the form

$a(t)=\mathrm{e}^{-\rho t}(e(t))^{1-\gamma}$,

obtaining a new boundary value problem for the function $e(t)$ of the form

$$
\begin{aligned}
& \frac{\mathrm{d} e(t)}{\mathrm{d} t}-H(t) e(t)+L(t)=0 \\
& e(T)=1,
\end{aligned}
$$


where $L(t)$ and $H(t)$ are as given in the statement of this Proposition. Since Eq. (22) is a linear, non-autonomous, first order ordinary differential equation, it clearly has an explicit solution of the form

$e(t)=\exp \left(-\int_{t}^{T} H(v) \mathrm{d} v\right)+\int_{t}^{T} \exp \left(-\int_{t}^{s} H(v) \mathrm{d} v\right) L(s) \mathrm{d} s$.

Therefore, we obtain that the solution of (20) is given by

$$
\begin{aligned}
a(t)= & \mathrm{e}^{-\rho t}\left(\exp \left(-\int_{t}^{T} H(v) \mathrm{d} v\right)\right. \\
& \left.+\int_{t}^{T} \exp \left(-\int_{t}^{s} H(v) \mathrm{d} v\right) L(s) \mathrm{d} s\right)^{1-\gamma} .
\end{aligned}
$$

To find a solution for the boundary value problem (21), we just note that this is again a linear, non-autonomous, first order differential equation and its solution is given by

$$
b(t)=\int_{t}^{T} i(s) \exp \left(-\int_{t}^{s}\left(r(v)+\eta_{k^{*}(v)}(v)\right) \mathrm{d} v\right) \mathrm{d} s .
$$

Combining (16) with (19), (23) and (24), we obtain that the optimal strategies in the case of CRRA utilities are then given by

$$
\begin{aligned}
& c^{*}(t, x)=\frac{1}{e(t)}(x+b(t)), \\
& \theta^{*}(t, x)=\frac{1}{1-\gamma} \frac{x+b(t)}{x} \xi \alpha(t), \\
& p_{k}^{*}(t, x)=\left\{\begin{array}{c}
\max \left\{0, \eta_{k}(t)((D(t)-1) x+D(t) b(t))\right\}, \\
\text { if } k=k^{*}(t) \\
0 \quad \text { otherwise, }
\end{array}\right.
\end{aligned}
$$

where $e(t)$ and $D(t)$ are as given in the statement of this proposition.

Remark 4.2. It can be seen from Proposition 4.1 that for a wage-earner with a sufficiently large wealth who is close to reaching retirement age, the optimal life-insurance selection and purchase is to buy no life-insurance at all. To see that this is indeed the case, note that as $t \rightarrow T$, we have that $e(t) \rightarrow 1$ and $b(t) \rightarrow 0$. Then, provided that $\lambda(t)<\eta_{k^{*}(t)}(t)$, we obtain that $D(t)<1$. Hence, for sufficiently large values of $x$, the term $(D(t)-1) x$ will dominate the term $D(t) b(t)$ and, thus, the quantity $\eta_{k^{*}(t)}(t)((D(t)-1) x+D(t) b(t))$ becomes negative.

\section{Conclusions}

We have studied the problem faced by a wage-earner with an uncertain lifetime in what concerns finding optimal insurance purchase, consumption and investment strategies among all of those available from an insurance market with multiple competing companies and a financial market consisting of one risk-free security and a fixed number of risky securities. We have used a form of the dynamic programming principle to reduce the stochastic optimal control problem under consideration here to one with a fixed planning horizon, stating also the corresponding $\mathrm{HJB}$ equation. We have determined the optimal strategies for the wage-earner in the special case of discounted CRRA utility functions. We prove that at each instant of time, such wage-earner either buys no life-insurance at all or buys life-insurance from the insurance company offering the smallest premium-payout ratio, abstaining from buying insurance from any other company.

\section{Acknowledgments}

We thank two anonymous referees for useful comments and suggestions. A.A. Pinto thanks the financial support of
LIAAD - INESC TEC and FCT Fundação para a Ciência e Tecnologia (Portuguese Foundation for Science and Technology) within project UID/EEA/50014/2013 and ERDF (European Regional Development Fund) through the COMPETE Program (operational program for competitiveness) and by National Funds through the FCT within Project "Dynamics, optimization and modelling", with reference PTDC/MAT-NAN/6890/2014. Alberto Adrego Pinto also acknowledges the financial support received through the project University of Porto/University of São Paulo and the Special Visiting Researcher Program (Bolsa Pesquisador Visitante Especial - PVE) "Dynamics, Games and Applications", with reference 401068/2014-5 (call: MEC/MCTI/CAPES/CNPQ/FAPS), at IMPA, Brazil. A.S. Mousa thanks the financial support of Birzeit University through the project with reference 240178. D. Pinheiro's research was supported by the PSC-Cuny research awards TRADA45-487 and TRADA-46-251, jointly funded by the Professional Staff Congress and the City University of New York.

\section{References}

Andersen, S., 1957. On the collective theory of risk in case of contagion between claims. Bull. Inst. Math. Appl. 12, 275-279

Asmussen, S., 1989. Risk theory in a Markovian environment. Scand. Actuar. J. 69-100.

Asmussen, S., 2000. Ruin Probabilities. World Scientific, Singapore.

Azevedo, N., Pinheiro, D., Weber, G.-W., 2014. Dynamic programming for a Markovswitching jump-diffusion. J. Comput. Appl. Math. 267, 1-19.

Bellman, R., 1952. On the theory of dynamic programming. Proc. Natl. Acad. Sci. USA 38 (8), 716-719.

Bellman, R., 1953. An introduction to the theory of dynamic programming, Rand Corporation Report, R-245.

Bellman, R., 1954. Dynamic programming and a new formalism in the calculus of variations. Proc. Natl. Acad. Sci. USA 40, 231-235.

Bellman, R., 1958. Dynamic programming and stochastic control process. Inf. Control 1, 228-239.

Blanchet-Scalliet, C., El Karoui, N., Jeanblanc, M., Martellini, L., 2008. Optimal investment decisions when time-horizon is uncertain. J. Math. Econom. 44 (11), $1100-1113$.

Bowers, N., Gerber, H., Hickman, J., Jones, D., Nesbitt, C., 1997. Actuarial Mathematics, second ed. Society of Actuaries, Schaumberg, Illinois.

Cramér, H., 1969. Historical review of filip Lundberg's works on risk theory. Scand. Actuar. J. 6-12.

Duarte, I., Pinheiro, D., Pinto, A.A., Pliska, S., 2012. Optimal life insurance purchase, consumption and investment on a financial market with multi-dimensional diffusive terms. Optimization 63 (11), 1737-1760.

Egídio dos Reis, A., 1993. How long is the surplus below zero? Insurance Math. Econom. 12, 23-38.

Fleming, W., Rishel, R., 1975. Deterministic and Stochastic Optimal Control. Springer-Verlag, New York, NY.

Fleming, W., Soner, H., 2006. Controlled Markov Processes and Viscosity Solutions, second ed. Springer-Verlag, New York, NY.

Florentin, J., 1961. Optimal control of continuous time, Markov, stochastic systems. J. Electron. Control 10, 473-488.

Florentin, J., 1962. Partial observability and optimal control. J. Electron. Control 13, 263-279.

Gerber, H., 1970. An extension of the renewal equation and its application in the collective theory of risk. Skand. Aktuarietidskr. 205-210.

Gerber, H., 1990. When does the surplus reach a given target? Insurance Math. Econom. 9, 115-119.

Gerber, H., Shiu, E., 1998. On the time value of ruin. N. Am. Actuar. J. 2 (1), 72-74

Hakansson, N., 1969. Optimal investment and consumption strategies under risk, an uncertain lifetime and insurance. Internat. Econom. Rev. 14, 132-152.

Hakansson, N., 1971. Optimal entrepreuneurial decisions in a completely stochastic environment. Manage. Sci. 17, 427-449.

Karatzas, I., Shreve, S., 1998. Methods of Mathematical Finance. Springer-Verlag, New York, NY.

Klugman, S., Panjer, H., Willmot, G., 2012. Loss Models: From Data to Decisions, fourth ed., Hoboken, NJ.

Kushner, H., 1962. Optimal stochastic control. IRE Trans. Automat. Control AC-7, $120-122$

Lundberg, F., 1903. Approximerad Framställning av Sannolikehetsfunktionen, Återförsäkering av Kollektivrisker. Almqvist \& Wiksell, Uppsala.

Lundberg, F., 1932. Some supplementary researches on the collective risk theory. Skand. Aktuarietidskr. 15, 137-158.

Merton, R., 1969. Lifetime portfolio selection under uncertainty: The continuous time case. Rev. Econ. Stat. 51 (3), 247-257.

Merton, R., 1971. Optimum consumption and portfolio rules in a continuous-time model. J. Econom. Theory 3 (4), 373-413.

Pliska, S., Ye, J., 2007. Optimal life insurance purchase and consumption/investment under uncertain lifetime. J. Bank. Finance 31, 1307-1319. 
Powers, M., 1995. A theory of risk, return and solvency. Insurance Math. Econom. 17 (2), 101-118

Richard, S., 1975. Optimal consumption, portfolio and life insurance rules for an uncertain lived individual in a continuous-time model. J. Financ. Econ. 2 (2), 187-203

Shenab, Y., Weib, J., 2014. Optimal investment-consumption-insurance with random parameters. Scand. Actuar. J.
Yaari, M., 1965. Uncertain lifetime, life insurance and the theory of the consumer. Rev. Econom. Stud. 32, 137-150.

Ye, J., 2006. Optimal life insurance purchase, consumption and portfolio under an uncertain life (Ph.D. thesis), University of Illinois at Chicago, Chicago, IL.

Yong, J., Zhou, X., 1999. Stochastic Controls: Hamiltonian Systems and HJB Equations. Springer-Verlag, New York, NY. 